\title{
Metformin prevents the development of monocrotaline-induced pulmonary hypertension by decreasing serum levels of big endothelin-1
}

\author{
TOMOHIKO YOSHIDA ${ }^{1}$, KATSUHIRO MATSUURA ${ }^{1}$, SEIJIROW GOYA ${ }^{1}$, DANFU MA ${ }^{1}$, KAZUMI SHIMADA ${ }^{1}$, \\ PITIPAT KITPIPATKUN $^{1}$, RYOSUKE NAMIKI ${ }^{1}$, AKIKO UEMURA ${ }^{1}$, KAZUHIKO SUZUKI $^{2}$ and RYOU TANAKA ${ }^{1}$
}

Departments of ${ }^{1}$ Veterinary Surgery and ${ }^{2}$ Veterinary Toxicology, Tokyo University of Agriculture and Technology, Tokyo 183-8509, Japan

Received July 2, 2019; Accepted January 16, 2020

DOI: $10.3892 /$ etm.2020.9278

\begin{abstract}
Pulmonary hypertension (PH) is a disease with poor prognosis, and it is characterized by the progressive elevation of pulmonary vascular resistance and pressure. Various factors are associated with the pathology of $\mathrm{PH}$, including AMP-activated protein kinase (AMPK) deficiency. The present study aimed to evaluate the therapeutic effect of metformin, an AMPK activator, in a monocrotaline (MCT)-induced PH rat model. Rats were randomly divided into the following three groups: i) Saline-injected group (sham group); ii) monocrotaline (MCT)-injected group (PH group); iii) MCT-injected and metformin-treated group (MT group). Four weeks following MCT injection, cardiac ultrasonography, invasive hemodynamic measurements, measurement of serum levels of big endothelin-1 (big ET-1) and histological analysis were performed to evaluate the effect of metformin treatment in PH. Pulmonary arterial pressure and serum big ET-1 concentrations were reduced in the MT group compared with the PH group. Medial wall thickness and wall area of the pulmonary arterioles in the MT group were decreased compared with the $\mathrm{PH}$ group. Comparing the right heart functional parameters among groups revealed that the acceleration time/ejection time ratio improved in the MT group compared with the $\mathrm{PH}$ group. Thus, the present study demonstrated the efficacy of metformin in an MCT-induced PH rat model and suggested that metformin may be a valuable, potential novel therapeutic for the treatment of $\mathrm{PH}$.
\end{abstract}

Correspondence to: Professor Ryou Tanaka, Department of Veterinary Surgery, Tokyo University of Agriculture and Technology, 3-5-8 Saiwai, Fuchu, Tokyo 183-8509, Japan

E-mail: ryo@vet.ne.jp

Key words: pulmonary hypertension, metformin, AMP-activated protein kinase, big endothelin-1, monocrotaline, echocardiography

\section{Introduction}

Pulmonary hypertension $(\mathrm{PH})$ is a rare disease that is characterized by the progressive elevation of pulmonary vascular resistance and pressure (1). $\mathrm{PH}$ is associated with poor prognosis, with a 5-year survival rate of $30 \%$ and estimated prevalence in different registries of between 15 and 26 cases per million population $>14$ years $(2,3)$. The progression of $\mathrm{PH}$ leads to right ventricular dysfunction and right-sided heart failure, which culminates in heart palpitations, dyspnea, syncope and ascites (4-7). The methods used to diagnose $\mathrm{PH}$ and evaluate the pathological conditions of patients with $\mathrm{PH}$ have dramatically improved due to development of new imaging equipment, including echocardiography, $\mathrm{X}$-ray, CT and MRI, in addition to the identification of novel biomarkers (8). In particular, prostacyclin analogues, phosphodiesterase-5 inhibitors, guanylate cyclase stimulators, prostacyclin receptor agonists and endothelin receptor antagonists, coupled with novel diagnostic and prognostic advances, have revolutionized treatment of PH (4). Although exercise tolerance, New York Heart Association (NYHA) functional class, hemodynamic parameters such as mean right atrial pressure (RAP) and cardiac index are all currently associated with $\mathrm{PH}$ prognosis, other factors are proposed to be involved (4). Of the number of factors that are hypothesized to contribute to $\mathrm{PH}$ pathology, pulmonary arterial endothelial cell dysfunction serves an important role. Endothelial cell dysfunction results from the overproduction of vasoconstrictors and proliferative factors, such as endothelin-1 (ET-1), and the reduced expression of vasodilators and antiproliferative factors, such as prostacyclin and nitric oxide (9-12). ET-1 is a vasoconstrictor peptide produced by vascular endothelial cells, which has been previously found to correlate positively with the severity PH (13-17). Therefore, ET-1 antagonists are frequently applied as a therapeutic agent for pulmonary hypertension (16). It has been reported that AMP-activated protein kinase (AMPK) deficiency promotes vascular endothelial cell dysfunction in association with ET-1 (18). AMPK is a serine/threonine kinase expressed in various tissues, and consists of a catalytic subunit, $\alpha$, and two regulatory subunits, $\beta$ and $\gamma$; it contributes to the maintenance of intracellular energy homeostasis, 
and it exhibits antiapoptotic effects in endothelial cells and anti-remodeling effects in vascular smooth muscle cells $(19,20)$. The anti-remodeling and antiapoptotic effects of AMPK are thought to attenuate the onset and progression of $\mathrm{PH}(18,21)$. Ibe et al (18) demonstrated that $\mathrm{AMPK} \alpha_{1}$ and $\mathrm{AMPK} \alpha_{2}$ had differential roles in the survival of pulmonary arterial smooth muscle cells under hypoxia and hypoxia-induced PH. In addition, Omura et al (22) demonstrated that long-term treatment of endothelial-specific AMPK-knockout mice with the AMPK activator, metformin, significantly attenuated hypoxia-induced $\mathrm{PH}$. Thus, metformin may represent a novel therapeutic agent for PH. The present study aimed to evaluate the therapeutic effects of metformin treatment in rats with monocrotaline (MCT)-induced PH using echocardiography and invasive pulmonary artery pressure (PAP) measurements, as well as evaluating biomarkers and histopathology.

\section{Materials and methods}

Animal studies. This study was approved by the Animal Experimental Subcommittee of Tokyo University of Agriculture and Technology (Tokyo, Japan). All animal experiments were conducted in accordance with the Regulations on Animal Experiments of Tokyo University of Agriculture and Technology, and with the Guide for the Care and Use of Laboratory Animals (23). A total of 36 male Wistar rats (age, 12 weeks; weight, 370-550 g; Oriental yeast, Co., Ltd.) were housed at $22^{\circ} \mathrm{C}, 40-70 \%$ relative humidity in a $21 \% \mathrm{O}_{2}$ room with a 12-h light/dark cycle and provided with free access to standard laboratory rat chow and water. Rats were randomly divided into the following three groups: i) Saline-injected group (sham; n=9); ii) MCT-injected group ( $\mathrm{PH} ; \mathrm{n}=19)$; iii) MCT-injected and metformin-treated group (MT; $n=8)$. Nine rats from the $\mathrm{PH}$ group died between the second and third weeks of drug administration due to drug-related complications: From the results of autopsy, the rats died from acute lung injury due to severe pulmonary arteritis $(24,25)$. MCT is an 11-member macrocyclic pyrrolizidine alkaloid that induces pulmonary arteritis in rats, which gradually progresses into $\mathrm{PH}$ (26-28). Previous studies have reported that the progression to $\mathrm{PH}$ occurs $\sim 4$ weeks following MCT administration (26-32). Rats in all groups, except for the sham group, were subjected to an intraperitoneal injection of $60 \mathrm{mg} / \mathrm{kg} \mathrm{MCT} \mathrm{(Wako} \mathrm{Pure} \mathrm{Chemical} \mathrm{Industries,} \mathrm{Ltd.)} \mathrm{to}$ induce $\mathrm{PH}$. MCT was dissolved in $1 \mathrm{M} \mathrm{HCl}$, and the $\mathrm{pH}$ was adjusted to 7.4 with $1 \mathrm{M} \mathrm{NaOH}$.

Metformin (Metformin hydrochloride; Pfizer, Inc.) was delivered orally (100 mg/kg/day) to the MT group through the drinking water, starting the day after MCT injection $(15,16)$. This solution was changed every day for 28 days. Four weeks after MCT injection, cardiac ultrasonography, invasive hemodynamic measurements and blood extraction were performed to evaluate the effects of the treatment. Rats were euthanized by exsanguination under anesthesia (the method of anesthesia was the same method as described for anesthesia for echocardiography) in accordance with the Regulations on Animal Experiments of Tokyo University of Agriculture and Technology and with the Guide for the Care and Use of Laboratory Animals. Following euthanasia, the lungs and hearts of all rats were excised for histopathological evaluation.
Right-sided heart functional evaluation by echocardiography. Echocardiography was performed under isoflurane anesthesia after $2.5 \mathrm{mg} / \mathrm{kg}$ butorphanol and $2.5 \mathrm{mg} / \mathrm{kg}$ midazolam were injected intramuscularly (33-36). The isoflurane concentration was adjusted between 1.0 and $1.5 \%$ to maintain the heart rate within the range of 300-350 beats/min (33). The thoraxes of the rats were shaved, and the rats were positioned in right and left lateral recumbency. Echocardiography was performed using a ProSound F75 PremierCV (Hitachi Aloka Medical, Ltd.), with a $7.5-\mathrm{MHz}$ transducer at a sweep speed of $300 \mathrm{~mm} / \mathrm{sec}$ and a sample gate of $1 \mathrm{~mm}$. The following echocardiographic parameters associated with $\mathrm{PH}$ were measured: From the parasternal short axis view at the mid-papillary muscle level, distance 1 (D1) was measured as the left ventricular minor axis diameter perpendicular to the septum, and distance 2 (D2) was measured as the left ventricular minor axis diameter parallel to the septum. The eccentricity index (EI) was defined as the ratio between D1 and D2 (EI=D1/D2). The tricuspid annular plane systolic excursion (TAPSE) was measured using M-mode across the tricuspid valve annulus at the right ventricle (RV) by positioning the echo cursor on the junction between the tricuspid valve plane and the RV-free wall $(\mathrm{Fw})$ using the apical four-chamber view. In the short-axis view at the aorta level, the ratio between the main pulmonary artery (MPA) diameter and the aortic diameter (Ao), MPA/Ao, and the ratio between the acceleration time (AT) and ejection time (ET), AT/ET, of the pulmonary artery (PA) flow velocity were measured $(25,27,30,37)$. The RV Tei index was calculated as the sum of the isovolumetric contraction time (ICT), and the isovolumetric relaxation time (IRT), divided by the ET, $(\mathrm{ICT}+\mathrm{IRT}) / \mathrm{ET}$, using the right parasternal RV outflow view $(13,14)$. Peak transtricuspid early diastolic wave velocity (E wave) was measured using the Doppler signals for the tricuspid inflow in the apical four-chamber view. The peak tissue Doppler tricuspid annular velocities at systole (Sm) and early diastole (Ea) in the apical four-chamber view were measured by focusing the sample volume of the tissue Doppler imaging on the $\mathrm{Fw}$ and the septal wall $(\mathrm{Mv})$, respectively, of the tricuspid valve annulus, and $\mathrm{E}$ wave/Ea was calculated $(25,38)$. Representative echocardiographic images of these measurements are presented in Fig. 1.

Hemodynamic measurements. Following the echocardiographic measurements, the rats were reoriented into a supine position to directly measure PAP under thoracotomy. The trachea of each rat was incised for tracheal tube insertion. Following intubation, the respiration mode was switched from spontaneous respiration to artificial respiration using an artificial ventilator (Harvard Apparatus). A catheter was inserted into the left carotid artery to monitor the invasive arterial pressure to measure heart rates, systolic blood pressure, mean blood pressure and diastolic blood pressure. The isoflurane concentration was carefully adjusted to maintain a mean blood pressure (MBP) $>60 \mathrm{mmHg}$, according to a pressure transducer. To measure the mean PAP (MPAP), the chest was opened at the fourth intercostal space and a catheter was directly inserted into the PA. The catheter was connected to a physiological pressure transducer and amplifier system (Life Scope BSM-5192; Nihon Kohden Co., Ltd.) to record pressure oscillations. PAP was recorded following the calibration 


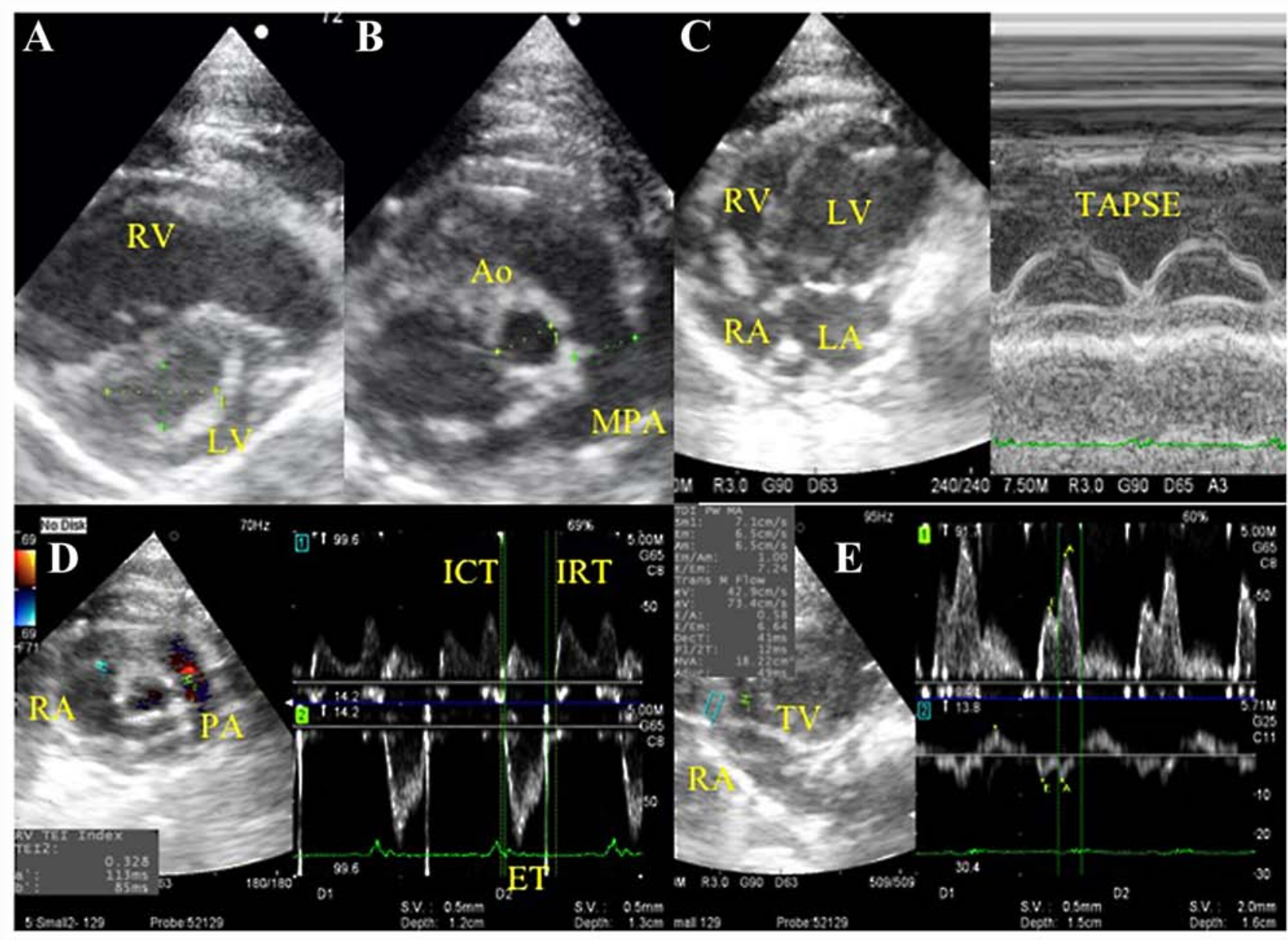

Figure 1. Representative echocardiogram images of right heart functional evaluation. (A-D) Representative images of the (A) eccentricity index from the parasternal short axis view, (B) MPA/Ao in the short-axis view at the aorta level, (C) TAPSE in the apical four-chamber view and (D) RV Tei index using the Dual Doppler system in the right parasternal RV outflow view. (E) Peak transtricuspid early diastolic wave velocity and tissue Doppler tricuspid annular velocities at systole and at early diastole were measured using the Dual Doppler system in the apical four-chamber view. Ao, aortic diameter; ET, ejection time; ICT, isovolumetric contraction time; IRT, isovolumetric relaxation time; LA, left atrium; LV, left ventricle; MPA, main pulmonary artery; PA, pulmonary artery; RA, right atrium; RV, right ventricle; TAPSE, tricuspid annular plane systolic excursion by M-mode imaging; TV, tricuspid valve.

and stabilization of the cardiac rhythm after anesthesia, as monitored using electrocardiogram leads and pulse oximetry.

Histological analysis. Lung tissue was fixed overnight in $10 \%$ buffered formalin at room temperature. Paraffin sections (5 $\mu \mathrm{m})$ were generated from each lobe, stained with hematoxylin \& eosin. All slide samples were washed with xylene for 5 min three times for deparaffinization before being dripped into methanol three times and washed in water for $1 \mathrm{~min}$. The slides were then shaken and stained with hematoxylin for $10 \mathrm{~min}$ at room temperature and eosin for $6 \mathrm{~min}$ at room temperature. All sections were analyzed with a light microscope to assess lung vascular pathology. Pulmonary arteries of 50-200 $\mu \mathrm{m}$ in diameter were evaluated by measuring arterial wall thickness at $\mathrm{x} 400$ magnification. For each rat, $\geq 10$ randomly selected circular or oval-shaped blood vessels were measured. The percentage medial wall thickness (MWT) of the arteries was calculated using the following formula: MWT $=[($ external diameter-internal diameter $) /$ external diameter] x100. The internal diameter was measured as the luminal diameter of the vessel, and the external diameter was measured as the total diameter of the vessel. The vessel area was also measured; wall area $(\mathrm{WA})=[$ (total vessel area-luminal area)/total vessel area] x100 $(26,31,37,39)$. Total vessel area and luminal area were measured using PCD software version 1.7 (FLOVEL Co., Ltd.).

Big ET-1 ELISA assay. Big ET-1 is a pre-cleavage pro-peptide of ET-1, which has a longer half-life compared with ET-1 and is therefore less likely to be inactivated than ET-1 (17). Blood samples $(4 \mathrm{ml})$ from the rats were collected from the carotid artery in tubes containing aprotinin. Serum was obtained following centrifugation $\left(1,200 \mathrm{x} \mathrm{g} ; 10 \mathrm{~min} ; 4^{\circ} \mathrm{C}\right)$, and it was stored at $-80^{\circ} \mathrm{C}$ until required. At the time of experimentation, frozen samples were thawed at room temperature and the extraction and concentration of Big ET-1 was performed using Sep Pak C18 cartridges (Waters Corporation). The samples were eluted through the Sep Pak C18 cartridges using $2 \mathrm{ml} 0.1 \%$ trifluoroacetic Acid $+60 \%$ acetonitrile (Wako Pure Chemical Industries, Ltd.). Serum big ET-1 concentrations were measured using a commercial rat big ET-1 ELISA kit (cat. no. 27168; Immuno-Biological Laboratories Co., Ltd.), according to the manufacturer's protocol. Because the primary antibody was immobilized on the plate, samples and exogenous standards were added to the plate to perform the primary reaction. After washing, horseradish peroxidase-labeled secondary 

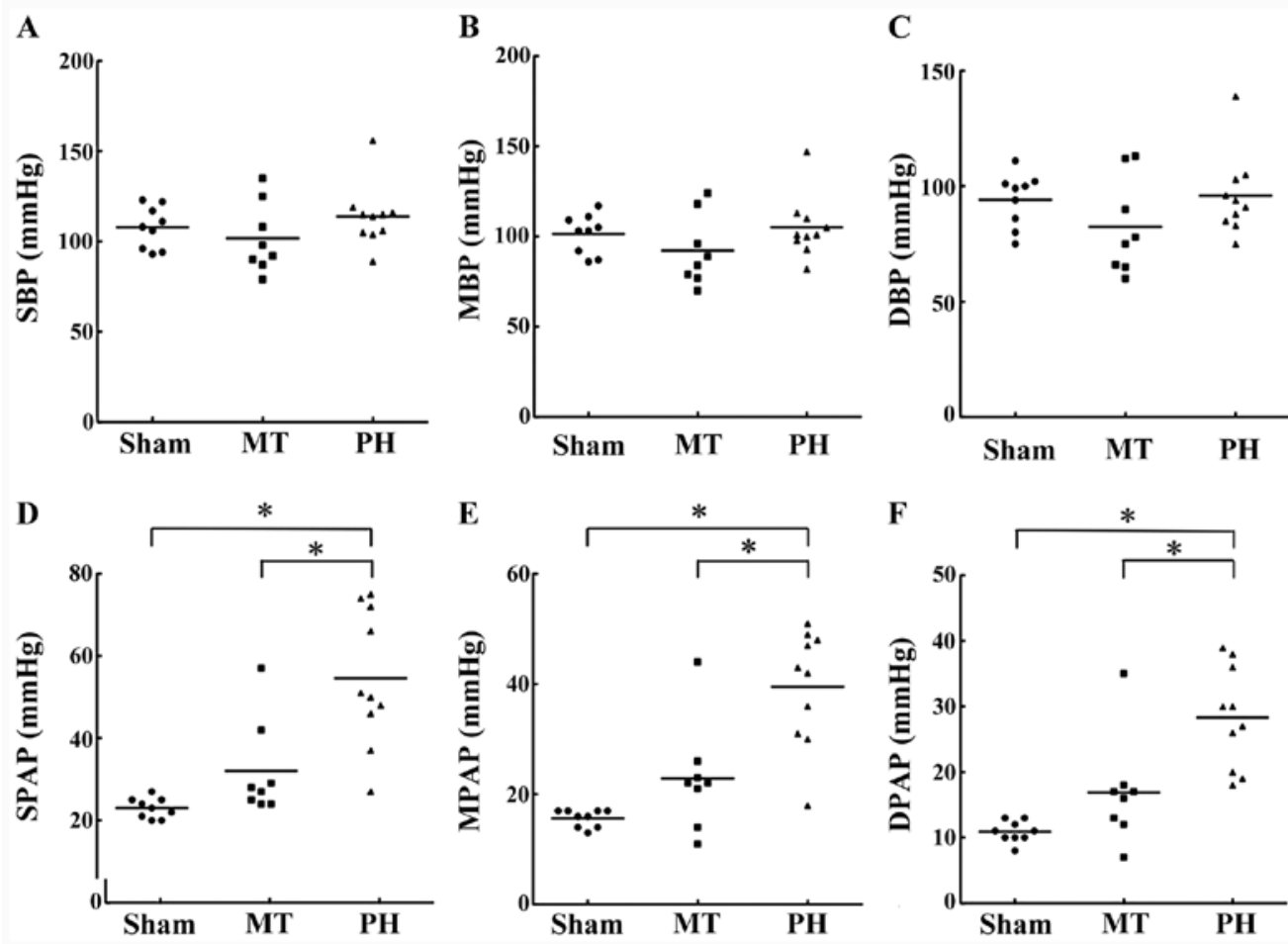

Figure 2. Blood pressure and pulmonary arterial pressure measurements in vivo. Measurements of (A) SBP, (B) MBP, (C) DBP, (D) SPAP, (E) MPAP and (F) DPAP in the sham group $(n=9)$, MT group $(n=8)$ and PH group $(n=10)$. Horizontal lines represent the median for each group. "P $<0.05$. DBP, diastolic blood pressure; DPAP, diastolic pulmonary artery pressure; MBP, mean blood pressure; MPAP, mean pulmonary artery pressure; MT, metformin treatment; $\mathrm{PH}$, pulmonary hypertension; SBP, systolic blood pressure; SPAP, systolic pulmonary artery pressure.

antibody was added to the plate to perform the secondary reaction. After the reaction, excess secondary antibody was washed away and 3,3',5'5'-tetramethylbenzidine was added to the wells. The resulting coloration was proportional to the concentration of rat big ET-1 in the sample, as measured using a microplate absorbance reader (Bio-Rad Laboratories, Inc.).

Statistical analysis. Data analysis was performed using Microsoft Excel 2016 (Microsoft Corporation), GraphPad Prism version 5.0a and InStat version 3.05 software (GraphPad Software, Inc.). All data are presented as mean \pm SD. Normality of all data was analyzed using a Bartlett's test. For normally distributed parameters, differences between groups were analyzed using a one-way ANOVA followed by post hoc analysis with Bonferroni correction. For non-parametric parameters, differences between groups were evaluated using a non-parametric Kruskal-Wallis test followed by post hoc analysis with Dunn's multiple comparison test. $\mathrm{P}<0.05$ was considered to indicate a statistically significant difference.

\section{Results}

Hemodynamic analysis of blood pressure and PAP. No significant differences were observed in the heart rates, systolic blood pressure, mean blood pressure, diastolic blood pressure, between the sham, PH and MT groups (Fig. 2A-C; Table I). Rats in the PH group developed severe PH compared with rats in the sham group, indicating the successful establishment of the PH model. Systolic PAP, mean PAP and diastolic PAP values in the $\mathrm{PH}$ group were significantly higher compared with the sham group, which were in turn reduced in the
MT group compared with the PH group. Therefore, metformin treatment could reduce PAP to levels comparable with that in the sham group (Fig. 2D-F).

Histopathological analysis of pulmonary arteries. Pulmonary vascular remodeling is central to the pathology of $\mathrm{PH}$, and the MWT and WA of PAs is an indicator of pulmonary vascular remodeling $(26,31,37,39)$. Histopathological analysis of the lung tissue demonstrated that rats in the $\mathrm{PH}$ group exhibited markedly thickened vascular walls within the pulmonary arterioles and increased proliferation of lung stromal cells (Fig. 3A and B). The pulmonary arteriole MWT (53.11 $\pm 8.25 \%)$ and WA $(75.62 \pm 7.40)$ were significantly increased in the $\mathrm{PH}$ group compared with the sham group (MWT, 20.26 $\pm 2.67 \%$; WA, 37.00 \pm 5.43 ) (Fig. 3C and D). Metformin treatment prevented PH-induced PA thickening in the MT group, with the pulmonary arteriolar MWT $(27.33 \pm 3.40 \%)$ and WA (49.28 \pm 6.10$)$ in the MT group being significantly reduced compared with the PH group. These results indicated that metformin treatment significantly inhibited the pathological pulmonary vascular remodeling induced by $\mathrm{PH}$.

Echocardiographic right heart functional evaluation. Comparisons of the right heart functional parameters between the groups are presented in Table I. No significant differences were identified between groups in EI, E wave, Ea Fw, Ea Mv, Sm, E/Ea Fw or E/Ea Mv values (Table I). The AT/ET ratio of both the $\mathrm{PH}$ and MT groups were significantly reduced compared with that in the sham group. Although the AT/ET ratio improved in the MT group compared with the $\mathrm{PH}$ group, it did not reach that of the sham group (Fig. 4A-C; 
Table I. Comparisons of the right heart functional parameters.

\begin{tabular}{|c|c|c|c|}
\hline Variables & Sham group & MT group & PH group \\
\hline Heart rate (beats/min) & $313.00 \pm 34.00$ & $301.00 \pm 34.00$ & $317.00 \pm 31.00$ \\
\hline Eccentricity index & $1.01 \pm 0.03$ & $1.04 \pm 0.09$ & $1.19 \pm 0.22$ \\
\hline Main pulmonary artery/aortic artery & $0.95 \pm 0.12$ & $0.97 \pm 0.05$ & $1.16 \pm 0.22^{\mathrm{a}}$ \\
\hline Accelerator time/ejection time & $0.43 \pm 0.05$ & $0.34 \pm 0.06^{\mathrm{a}}$ & $0.19 \pm 0.05^{\mathrm{a}, \mathrm{b}}$ \\
\hline Right ventricle Tei index & $0.36 \pm 0.03$ & $0.35 \pm 0.05$ & $0.47 \pm 0.07^{\mathrm{a}, \mathrm{b}}$ \\
\hline Peak transtricuspid early diastolic wave velocity (m/sec) & $0.69 \pm 0.16$ & $0.63 \pm 0.22$ & $0.62 \pm 0.16$ \\
\hline Early systole free wall $(\mathrm{cm} / \mathrm{sec})$ & $6.56 \pm 1.54$ & $6.86 \pm 1.78$ & $7.03 \pm 1.45$ \\
\hline Early systole septal wall (cm/sec) & $6.62 \pm 1.23$ & $5.53 \pm 1.13$ & $5.52 \pm 1.12$ \\
\hline Systole $(\mathrm{cm} / \mathrm{sec})$ & $5.65 \pm 0.89$ & $5.51 \pm 1.22$ & $5.71 \pm 1.38$ \\
\hline E wave/Ea free wall & $10.69 \pm 1.32$ & $9.41 \pm 3.43$ & $8.86 \pm 1.89$ \\
\hline E wave/Ea septal wall & $10.42 \pm 1.17$ & $11.42 \pm 4.00$ & $11.35 \pm 2.67$ \\
\hline Tricuspid annular plane systolic excursion (mm) & $3.02 \pm 0.20$ & $2.81 \pm 0.57$ & $2.27 \pm 0.51^{\mathrm{a}}$ \\
\hline
\end{tabular}

${ }^{\text {a }} \mathrm{P}<0.05$ vs. sham group; ${ }^{\mathrm{b}}<0.05$ vs. MT group. Sham group $(\mathrm{n}=9)$, MT group $(\mathrm{n}=8)$ and $\mathrm{PH}$ group $(\mathrm{n}=10)$. E, Peak transtricuspid early diastolic wave velocity; Ea, peak tissue Doppler tricuspid annular velocities at early diastole; MT, metformin; PH, pulmonary hypertension.

A

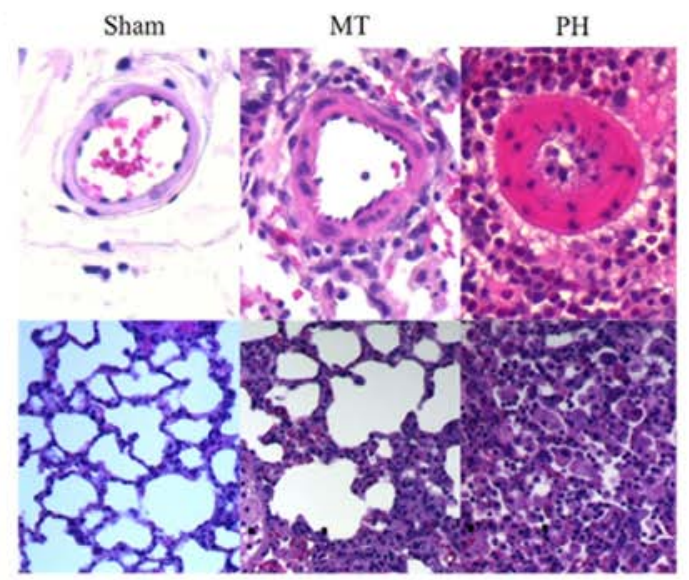

B

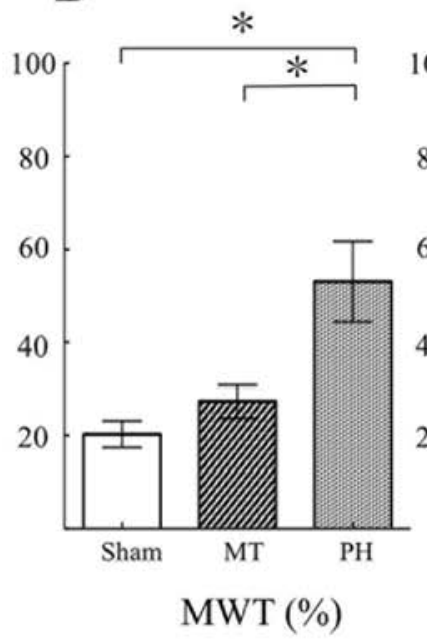

C

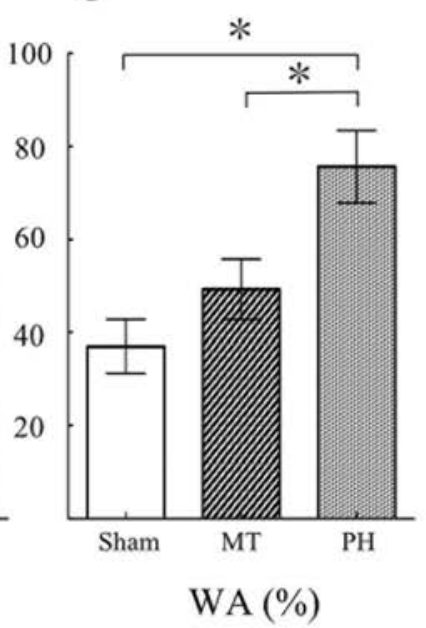

Figure 3. Histopathological analysis of pulmonary arterial remodeling four week after MCT injection. Hematoxylin \& eosin staining of the (A) small pulmonary artery (50-200 $\mu \mathrm{m}$ in diameter; magnification, $\mathrm{x} 400)$ and (B) lung stroma cells (magnification, $\mathrm{x} 400)$ in the sham group ( $=9$ ), MT group ( $=8$ ) and $\mathrm{PH}$ group $(\mathrm{n}=10)$. (C) Quantification of MWT and WA of pulmonary arteries from (A) and (B). Data are presented as mean \pm SD; ${ }^{2}<0.05$. MT, metformin treatment; MWT, medial wall thickness; $\mathrm{PH}$, pulmonary hypertension; WA, wall area.

Table I). There were significant differences in the MPA/Ao and TAPSE values between the sham and PH groups, but no significant differences were observed for these variables between the MT and PH groups (Table I). The RV Tei index, which is indicative of overall RV function $(13,14)$, presented significant differences between the sham and $\mathrm{PH}$ group, and significantly improved in the MT group relative to the PH group (Table I). This observation suggests that metformin treatment could improve echocardiographic parameters in patients with $\mathrm{PH}$.

Serum big ET-1 levels are upregulated in PH and decreased by metformin treatment. Big ET-1 concentrations were significantly increased in the PH group $(3.59 \pm 0.74 \mathrm{pg} / \mathrm{ml})$ compared with the sham group $(2.70 \pm 0.64 \mathrm{pg} / \mathrm{ml})$ (Fig. 5), but were reduced upon metformin treatment $(2.82 \pm 0.33 \mathrm{pg} / \mathrm{ml})$, with the MT group demonstrating significantly decreased serum levels of big ET-1 compared with the PH group. There was no significant difference reported between the MT group and the sham group (Fig. 5). These data suggest that blood big ET-1 levels were elevated in patients with $\mathrm{P}$, which were alleviated following metformin treatment.

\section{Discussion}

Although pulmonary vascular endothelial dysfunction and vascular remodeling are central to the pathology of $\mathrm{PH}$, the pathology of PH remains relatively unknown $(40,41)$. The present study focused on the AMPK activator, metformin, as 

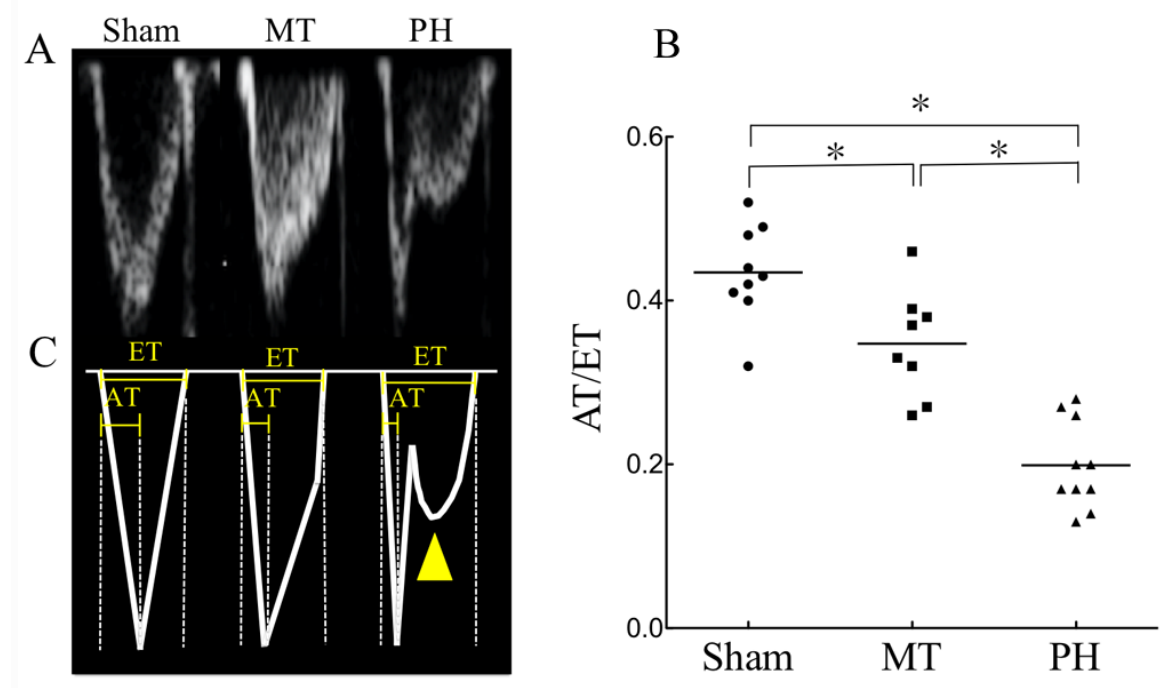

Figure 4. Evaluation of pulmonary artery flow. (A) Echocardiograph images of AT/ET in the sham group ( $\mathrm{n}=9$ ), MT group (n=8) and PH group (n=10). (B) Traces of the above images presented in (A). The images demonstrate the short flow AT in the MT and PH groups, and the flow reversal in the deceleration phase of pulmonary artery flow in the PH group (yellow arrow head). (C) AT/ET of pulmonary artery flow in each group. Each point represents an individual and the horizontal lines represent the median for each group; " $\mathrm{P}<0.05$. AT, acceleration time; ET, ejection time; MT, metformin treatment; PH, pulmonary hypertension.

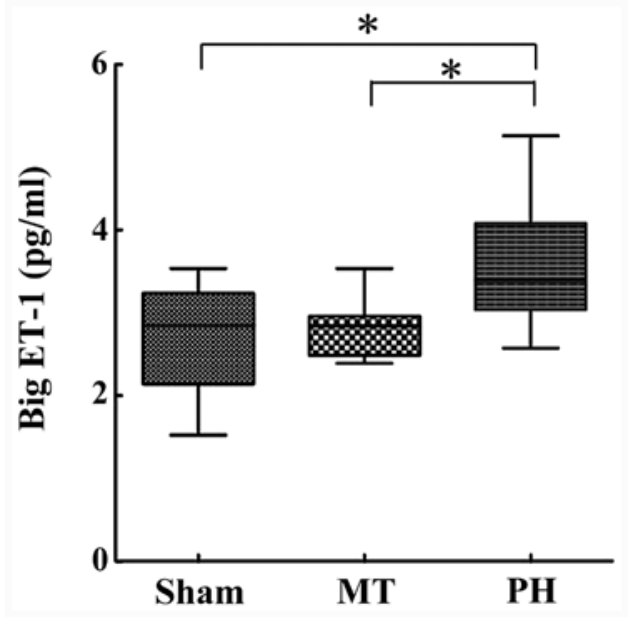

Figure 5. ELISA measured serum big ET-1 levels. Big ET-1 levels were determined in the sham $(\mathrm{n}=9), \mathrm{MT}(\mathrm{n}=8)$ and $\mathrm{PH}$ group $(\mathrm{n}=10)$. Data are presented as mean $\pm \mathrm{SD} ;{ }^{*} \mathrm{P}<0.05$. ET-1, endothelin-1; MT, metformin treatment; $\mathrm{PH}$, pulmonary hypertension.

AMPK is important for the maintenance of pulmonary vascular endothelial function $(22,42-44)$. Utilizing an MCT-induced PH rat model to evaluate the therapeutic effects of metformin in $\mathrm{PH}$, a relationship between metformin and serum ET-1, which is elevated in $\mathrm{PH}$, was determined.

Although the mechanism by which MCT causes PH remains unclear, pulmonary vascular remodeling, which progresses to cause pulmonary arterial vasculitis, is one of the possible mechanisms $(45,46)$. In the present study, histopathological analysis confirmed that MCT promoted pulmonary blood vessel remodeling and induced $\mathrm{PH}$. In addition to the MT group exhibiting significant decreases in MWT and WA values relative to the $\mathrm{PH}$ group, PAP was significantly reduced by metformin treatment; the mechanism by which metformin reduces PAP may be associated with its ability to inhibit vascular remodeling. In the context of $\mathrm{PH}$, metformin most likely maintains pulmonary vascular homeostasis by improving vascular endothelial cell function, as well as suppressing pulmonary vascular endothelial cell apoptosis and vascular smooth muscle cell remodeling (22).

In addition, metformin treatment decreased serum big ET-1 levels in PH. Big ET-1 is a vasoconstrictive and vasoproliferative factor produced by endothelial cells, and it is thought to potentiate pulmonary arterial vasoconstriction and remodeling in the context of PH $(14,17,47,48)$. These results suggested that the therapeutic effects of metformin in PH may be related to decreased peripheral big ET-1 concentrations. AMPK contributes to the maintenance of intracellular energy homeostasis, and it has been demonstrated to have antiapoptotic effects in endothelial cells and anti-remodeling effects in vascular smooth muscle cells (20-22). Tang et al (49) revealed that increased AMPK activity reduced ET-1 levels, by suppressing the $\mathrm{NF}-\kappa \mathrm{B} / \mathrm{NF}-\kappa \mathrm{B}$ inhibitor (I $\mathrm{B}) \alpha$ axis. NF- $\kappa \mathrm{B}$ forms part of a protein complex that regulates the immune response and cellular proliferation by controlling the expression of proinflammatory and pro-survival genes (50). AMPK suppresses $\mathrm{NF}-\kappa \mathrm{B}$ activity in a number of ways; thus, the therapeutic effects of metformin in the context of PH may be associated with the NF- $\kappa \mathrm{B} / \mathrm{I} \kappa \mathrm{B} \alpha$ axis. The study by Tang et al (49) suggested that dipeptidyl peptidase 4 inhibitor reduced ET-1 expression in the vascular endothelium by suppressing $\mathrm{NF}-\kappa \mathrm{B} / \mathrm{I} \kappa \mathrm{B} \alpha$ signaling through AMPK activation in diabetic rats. Therefore, reduced expression levels of big ET-1 in the serum of PH model rats following metformin treatment may be due to suppressed $\mathrm{NF}-\kappa \mathrm{B} / \mathrm{I} \kappa \mathrm{B} \alpha$ signaling.

In the present study, AT/ET was considered to be the most sensitive echocardiographic parameter for estimating the therapeutic effects of metformin on $\mathrm{PH}$. Numerous previous studies have reported that $\mathrm{AT} / \mathrm{ET}$ is decreased in patients with PH $(25,27,30,37)$. Similarly, the results obtained in this 
study demonstrated that AT/ET was significantly reduced in the $\mathrm{PH}$ model rats compared with the other groups. In addition, AT/ET reflected the therapeutic effect of metformin between MT group and PH group. The RV Tei index is an echocardiographic parameter that reflects the severity of PH $(51,52)$. The RV Tei index was significantly higher in the PH group compared to the other groups; however, because no significant difference was observed between the MT group and the sham group, it indicated that the RV Tei index may not be as sensitive as AT/ET in evaluating PH severity. Both AT/ET and RV Tei index are considered to be more useful compared with TAPSE and MPA/PA values for the evaluation of PH severity in the present study. Although clear evidence remains to be lacking, AT/ET and RV Tei index appear to be sensitive because they can capture changes in blood flow with higher degrees of sensitivity in the present study.

Nonetheless, there are limitations to this study and the results should be interpreted with caution. Metformin was administered prophylactically prior to MCT induction of $\mathrm{PH}$; therefore, the therapeutic effects of metformin following the onset of $\mathrm{PH}$ remain unclear. Further studies regarding the anti-remodeling effects of metformin are also necessary, and the detailed mechanisms underlying these effects must be investigated at the molecular level. Furthermore, big ET-1 concentrations and expression of preproET-1 mRNA, which encodes the precursor protein of big ET-1 in tissues, in pulmonary vascular endothelial tissue should be examined to identify the origin of serum big ET-1, in addition to measuring other biomarkers associated with PH. Additionally, it remains unclear whether the dose of metformin used in this study was within the physiological range permitted for humans; as such, the dose of metformin should also be investigated in further studies.

To the best of our knowledge, this is the first study to report that metformin prevented the development of MCT-induced PH by decreasing the serum ET-1 concentration. The possibility of this finding being associated with the AMPK pathway warrants further future study. Multiple previous studies have reported that the homeostatic improvement of vascular endothelial cells by AMPK was effective for the treatment of PH $(20,22,43)$. However, in addition to this mechanism, it can be hypothesized that the increased expression of ET-1, produced in vascular endothelial cells, is suppressed by improved homeostasis of vascular endothelial cells following AMPK activation.

In conclusion, the present study demonstrated that early-stage metformin treatment significantly attenuated the development of MCT-induced PH, hemodynamically and histopathologically, which may have been related to suppression of serum big ET-1. These results suggested that metformin may be a putative therapeutic strategy for $\mathrm{PH}$, but further studies are required to understand the detailed mechanisms underlying these effects.

\section{Acknowledgements}

The authors would like to thank the Fujifilm Monolis Co., Ltd. (Tokyo, Japan) for technical assistance with experiments and providing the facilities.

\section{Funding}

No funding was received.

\section{Availability of data and materials}

The datasets used and/or analyzed during the present study are available from the corresponding author on reasonable request.

\section{Authors' contributions}

TY designed the study and wrote the initial draft of the manuscript. DM, KSh, RN and PK maintained the animals. KM contributed to analysis and interpretation of data. KSu prepared tissue specimens and provided advise for the histopathological studies. SG, AU and RT contributed to data interpretation and critically reviewed the manuscript. All authors read and approved the final version of the manuscript.

\section{Ethics approval and consent to participate}

All experiments were conducted in accordance with the Regulations on Animal Experiments of Tokyo University of Agriculture and Technology (Tokyo, Japan) and with the Guide for the Care and Use of Laboratory Animals. This study was approved by the Animal Experimental subcommittee of Tokyo University of Agriculture and Technology (permit no. 30-88).

\section{Patient consent for publication}

Not applicable.

\section{Competing interests}

The authors declare that they have no competing interests.

\section{References}

1. D'Alonzo GE, Barst RJ, Ayres SM, Bergofsky EH, Brundage BH, Detre KM, Fishman AP, Goldring RM, Groves BM, Kernis JT, et al: Survival in patients with primary pulmonary hypertension: Results from a national prospective registry. Ann Intern Med 115: 343-349, 1991.

2. Gall H, Felix JF, Schneck FK, Milger K, Sommer N, Voswinckel R, Franco OH, Hofman A, Schermuly RT, Weissmann N, et al: The giessen pulmonary hypertension registry: Survival in pulmonary hypertension subgroups. J Heart Lung Transplant 36: 957-967, 2017.

3. Escribano-Subias P, Blanco I, López-Meseguer M, Lopez-Guarch CJ, Roman A, Morales P, Castillo-Palma MJ, Segovia J, Gómez-Sanchez MA and Barberà JA; REHAP investigators: Survival in pulmonary hypertension in Spain: Insights from the Spanish registry. Eur Respir J 40: 596-603, 2012.

4. Galiè N, Humbert M, Vachiery JL, Gibbs S, Lang I, Torbicki A, Simonneau G, Peacock A, Noordegraaf AV, Beghetti M, et al: 2015 ESC/ERS guidelines for the diagnosis and treatment of pulmonary hypertension: The joint task force for the diagnosis and treatment of pulmonary hypertension of the european society of cardiology (ESC) and the european respiratory society (ERS): Endorsed by: Association for european paediatric and congenital cardiology (AEPC), international society for heart and lung transplantation (ISHLT). Eur Heart J 37: 67-119, 2015.

5. Barberà JA, Román A, Gómez-Sánchez MÁ, Blanco I, Otero R, López-Reyes R, Otero I, Pérez-Peñate G, Sala E and Escribano P: Guidelines on the diagnosis and treatment of pulmonary hypertension: Summary of recommendations. Arch Bronconeumol 54: 205-215, 2018 (In English, Spanish). 
6. Barst RJ, McGoon M, Torbicki A, Sitbon O, Krowka MJ, Olschewski H and Gaine S: Diagnosis and differential assessment of pulmonary arterial hypertension. J Am Coll Cardiol 43 : S40-S47, 2004

7. Lai YC, Potoka KC, Champion HC, Mora AL and Gladwin MT: Pulmonary arterial hypertension: The clinical syndrome. Circ Res 115: 115-130, 2014.

8. Gupta H, Ghimire G and Naeije R: The value of tools to assess pulmonary arterial hypertension. Eur Respir Rev 20: 222-235, 2011.

9. Chester AH, Yacoub MH and Moncada S: Nitric oxide and pulmonary arterial hypertension. Glob Cardiol Sci Pract 2017: 14, 2017.

10. Cracowski JL and Leuchte HH: The potential of biomarkers in pulmonary arterial hypertension. Am J Cardiol 110 (Suppl 6): S32-S38, 2012

11. Shao D, Park JE and Wort SJ: The role of endothelin-1 in the pathogenesis of pulmonary arterial hypertension. Pharmacol Res 63: 504-511, 2011.

12. Wang X, Xu Q, Li T, Rong Y, Hong W, Huang Y and Guo X Intratracheal administration of isosorbide dinitrate improves pulmonary artery pressure and ventricular remodeling in a rat model of heart failure following myocardial infarction. Exp Ther Med 14: 1399-1408, 2017

13. Montani D, Souza R, Binkert C, Fischli W, Simonneau G Clozel $\mathrm{M}$ and Humbert $\mathrm{M}$ : Endothelin-1/endothelin-3 ratio: A potential prognostic factor of pulmonary arterial hypertension. Chest 131: 101-108, 2007.

14. Simon M, Battistini B, Kim YJ and Tsang J: Plasma levels of endothelin-1, big endothelin-1 and thromboxane following acute pulmonary air embolism. Respir Physiol Neurobiol 138: 97-106, 2003.

15. Satwiko MG, Ikeda K, Nakayama K, Yagi K, Hocher B, Hirata K and Emoto N: Targeted activation of endothelin-1 exacerbates hypoxia-induced pulmonary hypertension. Biochem Biophys Res Commun 465: 356-362, 2015.

16. Vizza CD, Letizia C, Badagliacca R, Poscia R, Pezzuto B Gambardella C, Nona A, Papa S, Marcon S, Mancone M, et al: Relationship between baseline ET-1 plasma levels and outcome in patients with idiopathic pulmonary hypertension treated with bosentan. Int J Cardiol 167: 220-224, 2013.

17. Fukumoto S, Hanazono K, Miyasho T, Endo Y, Kadosawa T, Iwano $\mathrm{H}$ and Uchide $\mathrm{T}$ : Serum big endothelin-1 as a clinical marker for cardiopulmonary and neoplastic diseases in dogs. Life Sci 118: 329-332, 2014.

18. Ibe JCF, Zhou Q, Chen T, Tang H, Yuan JXJ, Raj JU and Zhou G: Adenosine monophosphate-activated protein kinase is required for pulmonary artery smooth muscle cell survival and the development of hypoxic pulmonary hypertension. Am J Respir Cell Mol Biol 49: 609-618, 2013.

19. Evans AM, Hardie DG, Peers C and Mahmoud A: Hypoxic pulmonary vasoconstriction: Mechanisms of oxygen-sensing. Curr Opin Anaesthesiol 24: 13, 2011.

20. Wu Y, Liu L, Zhang Y, Wang G, Han D, Ke R, Li S, Feng W and Li M: Activation of AMPK inhibits pulmonary arterial smooth muscle cells proliferation. Exp Lung Res 40: 251-258, 2014.

21. Agard C, Rolli-Derkinderen M, Dumas-de-La-Roque E, Rio M, Sagan C, Savineau JP, Loirand G and Pacaud P: Protective role of the antidiabetic drug metformin against chronic experimental pulmonary hypertension. Br J Pharmacol 158: 1285-1294, 2009.

22. Omura J, Satoh K, Kikuchi N, Satoh T, Kurosawa R, Nogi M, Otsuki T, Kozu K, Numano K, Suzuki K, et al: Protective roles of endothelial AMP-activated protein kinase against hypoxia-induced pulmonary hypertension in mice. Circ Res 119: 197-209, 2016.

23. National Researrch Council: Guide for the Care and Use of Laboratory Animals. 8th edition. National Academies Press. Washingthon DC, 2010.

24. Gomez-Arroyo JG, Farkas L, Alhussaini AA, Farkas D, Kraskauskas D, Voelkel NF and Bogaard HJ: The monocrotaline model of pulmonary hypertension in perspective. Am J Physiol Lung Cell Mol Physiol 302: L363-L369, 2012.

25. Nakata TM, Tanaka R, Yoshiyuki R, Fukayama T, Goya S and Fukushima R: Effects of single drug and combined short-term administration of sildenafil, pimobendan, and nicorandil on right ventricular function in rats with monocrotaline-induced pulmonary hypertension. J Cardiovasc Pharmacol 65: 640, 2015.

26. Bogdan S, Seferian A, Totoescu A, Dumitrache-Rujinski S, Ceausu M, Coman C, Ardelean CM, Dorobantu M and Bogdan M Sildenafil reduces inflammation and prevents pulmonary arterial remodeling of the monocrotaline-induced disease in the Wistar rats. Maedica 7: 109, 2012.
27. Wang Y, Tian W, Xiu C, Yan M, Wang S and Mei Y: Urantide improves the structure and function of right ventricle as determined by echocardiography in monocrotaline-induced pulmonary hypertension rat model. Clin Rheumatol 38: 29-35, 2019.

28. Tawa M, Furukawa T, Tongu H, Sugihara M, Taguwa S, Yamanaka M, Yano Y, Matsumori H, Kitada R, Sawano T, et al: Stimulation of nitric oxide-sensitive soluble guanylate cyclase in monocrotaline-induced pulmonary hypertensive rats. Life Sci 203: 203-209, 2018.

29. Breitling S, Krauszman A, Parihar R, Walther T, Friedberg MK and Kuebler WM: Dose-dependent, therapeutic potential of angiotensin-(1-7) for the treatment of pulmonary arterial hypertension. Pulm Circ 5: 649-657, 2015.

30. Pacagnelli FL, Sabela AKD, Mariano TB, Ozaki GAT, Castoldi RC, Carmo EM, Carvalho RF, Tomasi C, Okoshi K and Vanderlei LCM: Fractal dimension in quantifying experimental-pulmonary-hypertension-induced cardiac dysfunction in rats. Arq Bras Cardiol 107: 33-39, 2016

31. Karasu-Minareci E, Ozbudak IH, Ozbilim G and Sadan G: Acute effects of vardenafil on pulmonary artery responsiveness in pulmonary hypertension. ScientificWorldJournal 2012: 718279, 2012.

32. Bae HK, Lee H, Kim KC and Hong YM: The effect of sildenafil on right ventricular remodeling in a rat model of monocrotaline-induced right ventricular failure. Korean J Pediatr 59: 262-270, 2016.

33. Tsukamoto A, Uchida K, Maesato S, Sato R, Kanai E and Inomata T: Combining isoflurane anesthesia with midazolam and butorphanol in rats. Exp Anim 65: 223-230, 2016.

34. Aimbire F, Penna SC, Rodrigues KC, Lopes-Martins RAB and Serté JAA: Effect of hydroalcoholic extract of zingiber officinalis rhizomes on LPS-induced rat airway hyperreactivity and lung inflammation. Prostaglandins Leukot Essent Fatty Acids 77: 129-138, 2007.

35. Albrecht M, Henke J, Tacke S, Markert M and Guth B: Effects of isoflurane, ketamine-xylazine and a combination of medetomidine, midazolam and fentanyl on physiological variables continuously measured by telemetry in wistar rats. BMC Vet Res 198: 10-23, 2014.

36. Gades NM, Danneman PJ, Wixson SK and Tolley EA: The magnitude and duration of the analgesic effect of morphine, butorphanol, and buprenorphine in rats and mice. Contemp Top Lab Anim Sci 39: 8-13, 2000.

37. Lee JH, Park BK, Oh KS, Yi KY, Lim CJ, Seo HW and Lee BH: A urotensin II receptor antagonist, KR36676, decreases vascular remodeling and inflammation in experimental pulmonary hypertension. Int Immunopharmacol 40: 196-202, 2016.

38. Kimura K, Daimon M, Morita H, Kawata T, Nakao T, Okano T, Lee SL, Takenaka K, Nagai R, Yatomi Y and Komuro I: Evaluation of right ventricle by speckle tracking and conventional echocardiography in rats with right ventricular heart failure. Int Heart J 56: 349-353, 2015.

39. Itoh T, Nagaya N, Fujii T, Iwase T, Nakanishi N, Hamada K, Kangawa $\mathrm{K}$ and Kimura $\mathrm{H}$ : A combination of oral sildenafil and beraprost ameliorates pulmonary hypertension in rats. Am J Respir Crit Care Med 169: 34-38, 2004.

40. Hirose S, Hosoda Y, Furuya S, Otsuki T and Ikeda E: Expression of vascular endothelial growth factor and its receptors correlates closely with formation of the plexiform lesion in human pulmonary hypertension. Pathol Int 50: 472-479, 2000

41. Tuder RM, Groves B, Badesch DB and Voelkel NF: Exuberant endothelial cell growth and elements of inflammation are present in plexiform lesions of pulmonary hypertension. Am J Pathol 144: 275-285, 1994.

42. Dean A, Nilsen M, Loughlin L, Salt IP and MacLean MR: Metformin reverses development of pulmonary hypertension via aromatase inhibition. Hypertension 68: 446-454, 2016.

43. Zhai C, Shi W, Feng W, Zhu Y, Wang J, Li S, Yan X, Wang Q, Zhang Q, Chai L, et al: Activation of AMPK prevents monocrotaline-induced pulmonary arterial hypertension by suppression of NF- $\kappa$ B-mediated autophagy activation. Life Sci 208: 87-95, 2018.

44. Hattori Y, Suzuki K, Hattori S and Kasai K: Metformin inhibits cytokine-induced nuclear factor kappaB activation via AMP-activated protein kinase activation in vascular endothelial cells. Hypertension 47: 1183-1188, 2006.

45. Lalich $\mathbf{J}$ and Merkow L: Pulmonary arteritis produced in rats by feeding crotalaria spectabilis. Lab Invest 10: 744-750, 1961.

46. Yamaguchi K, Kanai Y, Asano K, Takasugi T, Tanaka T, Yasuoka $M$ and Hosoda Y: Temporal alterations of endothelial-vasodilator functions in lung injury induced by monocrotaline. Respir Physiol 107: 47-58, 1997. 
47. Rubens C, Ewert R, Halank M, Wensel R, Orzechowski HD, Schultheiss HP and Hoeffken G: Big endothelin-1 and endothelin-1 plasma levels are correlated with the severity of primary pulmonary hypertension. Chest 120: 1562-1569, 2001.

48. Stangl K, Dschietzig T, Richter C, Laule M, Stangl V, Tanis E, Baumann G and Felix SB: Pulmonary release and coronary and peripheral consumption of big endothelin and endothelin-1 in severe heart failure: Acute effects of vasodilator therapy. Circulation 102: 1132-1138, 2000.

49. Tang ST, Su H, Zhang Q, Tang HQ, Wang CJ, Zhou Q, Wei W, Zhu HQ and Wang Y: Sitagliptin inhibits endothelin-1 expression in the aortic endothelium of rats with streptozotocin-induced diabetes by suppressing the nuclear factor $-\kappa \mathrm{B} / \mathrm{I} \kappa \mathrm{B} \alpha$ system through the activation of AMP-activated protein kinase. Int J Mol Med 37: 1558-1566, 2016.
50. Barnes PJ and Karin M: Nuclear factor- $\kappa$ B: A pivotal transcription factor in chronic inflammatory diseases. N Engl J Med 336: 1066-1071, 1997.

51. Tei C, Dujardin KS, Hodge DO, Bailey KR, McGoon MD, Tajik AJ and Seward SB: Doppler echocardiographic index for assessment of global right ventricular function. J Am Soc Echocardiogr 9: 838-847, 1996.

52. Seyfarth HJ, Pankau H, Hammerschmidt S, Schauer J, Wirtz H and Winkler J: Bosentan improves exercise tolerance and Tei index in patients with pulmonary hypertension and prostanoid therapy. Chest 128: 709-713, 2005.

This work is licensed under a Creative Commons Attribution-NonCommercial-NoDerivatives 4.0 International (CC BY-NC-ND 4.0) License. 